\title{
Self-assembly of 3D Graphene and Its Superior Adsorption Ability for Heavy Oil
}

\author{
Bo ZHOU ${ }^{1, a,{ }^{*},}$, Yan WU ${ }^{1, b}$, Yao-Qun HUANG ${ }^{1, c}$, Shu WANG ${ }^{1, d}$, Guo-An ZHA ${ }^{1, e}$ \\ ${ }^{1}$ Engineering Research Academy of Graphite New Materials, Heilongjiang University of Science \\ and Technology, Harbin, 150022, PR China \\ aapenbox@126.com, ’wuyanusth@163.com, chuangyaoqun@126.com, d894201470@qq.com, \\ ewojiaoguoan@163.com \\ ${ }^{*}$ Corresponding author
}

Keywords: 3D Graphene, Self-assembly, Adsorption.

\begin{abstract}
Three-dimensional graphene (3DG) samples were prepared using template-free solventthermal synthesis route with graphene oxide $(\mathrm{GO})$ as a precursor. With the GO concentration $\left(C_{\mathrm{GO}}\right)$ increasing gradually from $4 \mathrm{mg} / \mathrm{mL}$ to $10 \mathrm{mg} / \mathrm{mL}$, the morphology of the 3DG evolves from nanoparticle to $3 \mathrm{DG}$ monolith material. The $3 \mathrm{DG}$ bulk is obtained when the $C_{\mathrm{GO}}$ increased to 8 $\mathrm{mg} / \mathrm{mL}$ and $10 \mathrm{mg} / \mathrm{mL}$. Absorption tests of various liquids have been performed, indicating ome outstanding features of 3DG efficient and quick absorption of oils and organic solvents.
\end{abstract}

\section{Introduction}

Adsorption is commonly considered to be a fast and relatively cost-effective technology for water treatment [1]. It normally occurs via both physical and chemical pathways, like activated carbon, which offers the advantage of its porous structure and surface-modified functionalities. Similarly, graphene, consisting of 2D hexagonal lattices of $\mathrm{sp} 2$ carbon atoms covalently bonded, has been theorized to have a huge specific surface area (over $2600 \mathrm{~m}^{2} \mathrm{~g}^{-1}$ ). On the other hand, the applications of graphene-based materials in energy, environment, sensing and biological fields often need assembling 2D graphene sheets into 3D architectures [2-5]. The 3D structures would provide graphene materials with high specific surface areas, strong mechanically strengths and fast mass and electron transport kinetics due to the combination of 3D porous structure and the excellent intrinsic properties of graphene. The rationally designed macroscopic architectures may offer additional fundamental scientific opportunities, such as the collective optical, electronic, and mechanical properties.

In the past few years, 3DG quickly drew extensive research interest, especially in environmental treatment [6,7]. This graphene-based monolith usually has a low-density porous structure which allows diffusion of ions and molecules throughout. More importantly, compared to graphene, 3DG can effectively avoid layer-layer aggregation and retain the large specific surface, not to mention the convenience it brought to separation and recycling processes. Increasing studies have shown the good adsorption capability of 3DG for pollutants such as heavy metal ions, organic solvents and dyes. For wider applications of 3DG in environmental treatment, a feasible synthetic method appropriate for adsorption is needed.

So far, two synthetic methods of 3DG have been developed - template growth and free-standing. The template growth method utilizes chemical vapor deposition (CVD) to grow graphene structures directly on the sacrificed template, which requires precise control of temperature and atmosphere. In comparison, self-assembly, one of freestanding method, is attracting more attention for its ease of operation. Herein, we would like to use the self-assembly method to make graphene sheets into 3D macroscopic structures, and its adsorption ability for heavy oil would be discussed.

\section{Experimental Section}

\section{Materials}

All chemicals were reagent-grade and used without further purification. Graphite, Sulfuric acid $\left(\mathrm{H}_{2} \mathrm{SO}_{4}\right)$, hydrochloric acid $(\mathrm{HCl})$, hydrogen peroxide $\left(\mathrm{H}_{2} \mathrm{O}_{2}\right)$, potassium permanganate $\left(\mathrm{KMnO}_{4}\right)$, 
and sodium nitrate $\left(\mathrm{NaNO}_{3}\right)$ were purchased from Sinopharm Chemical Reagent Co., Ltd., of China. The soybean was from supermarket, and the engie oil and diesel oil were purchased from China petroleum and gas station. All solutions were prepared using deionized (DI) water.

\section{Preparation of self-assembled 3D Graphene}

Graphene oxide (GO) was prepared by the improved Hummer method. A $30 \mathrm{~mL}$ portion of 4, 6 , 8 or $10 \mathrm{mg} / \mathrm{mL}$ homogeneous GO aqueous dispersion was sealed in a $50 \mathrm{~mL}$ Teflon-lined autoclave and maintained at $150 \sim 200{ }^{\circ} \mathrm{C}$ for $12 \mathrm{~h}$. Then the autoclave was naturally cooled to room temperature and the as-prepared samples were taken out with a tweezer and blotted with a filter paper to remove surface adsorbed water. The 3DG samples were dried in a freeze dryer, with an initial shelf temperature of $25{ }^{\circ} \mathrm{C}$ and a condenser temperature of $-80{ }^{\circ} \mathrm{C}$, and an ultimate $5 \mu$ bar vacuum was applied to sublime the icefreeze-dried samples.

\section{Adsorption Procedure}

Adsorption experiments were performed at room temperature. Absorption of soybean oil in 3DG at intervals of $1 \mathrm{~min} .2 \mathrm{~mL}$ heavy oil floating on water, the dosage of 3DG macrostructure was 30 $\mathrm{mg}$ for each adsorption experiment. We use activated carbon (AC) for comparison study. The efficiency of oil absorption can be referred to as weight gain, i.e., wt $\%$, defined as the weight of absorbed oil per unit weight of 3DG.

\section{Results and Discussion}

The typical 3DG can be easily prepared by heating $8 \mathrm{mg} / \mathrm{mL}$ of homogeneous graphene oxide (GO) aqueous dispersion sealed in a Teflon-lined autoclave at $180{ }^{\circ} \mathrm{C}$ for $12 \mathrm{~h}$ (Fig. 1a). The as-prepare 3DG with a diameter around $1.5 \mathrm{~cm}$ and $3.2 \mathrm{~cm}$ high (Fig. 1b). The 3DG has a well-defined and interconnected 3D porous network as imaged by scanning electron microscopy (SEM) (Fig.1c). The pore sizes of the network are in the range of sub-micrometer to several micrometers and the pore walls consist of thin layers of stacked graphene sheets. The partial overlapping or coalescing of flexible graphene sheets resulted in the formation of physical cross-linking sites of the framework of the 3DG (Fig. 1c). Thus, the inherent flexibility of graphene sheets is a crucial property for constructing the 3D macrostructures.

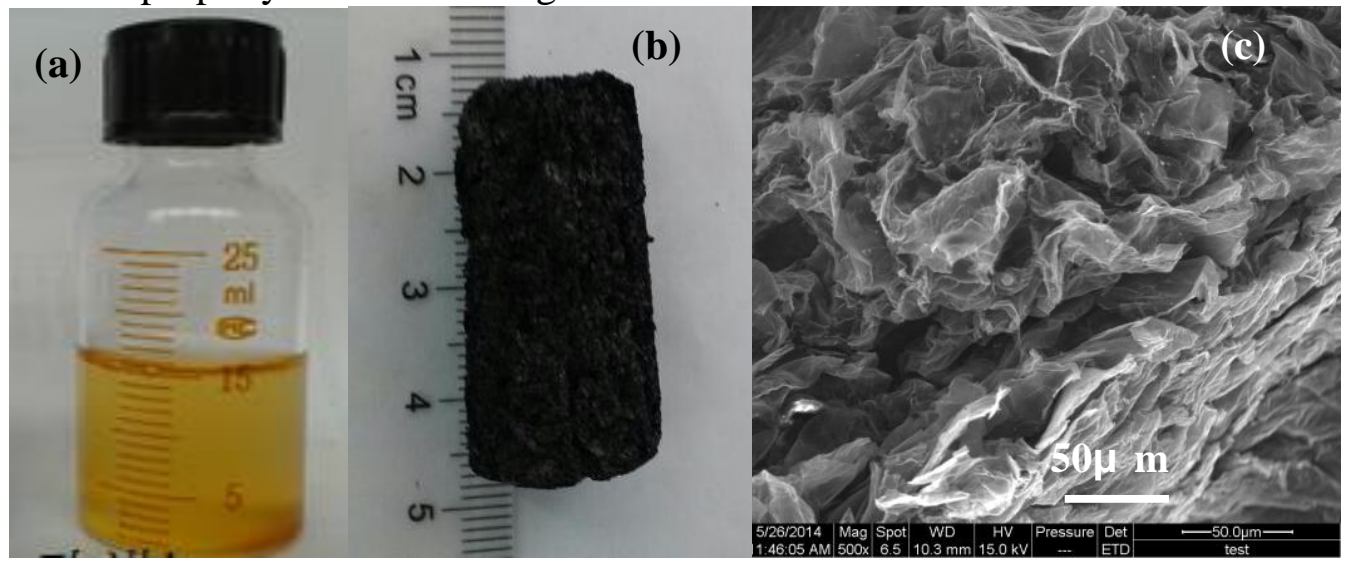

Fig.1 (a) Photographs of a $8 \mathrm{mg} / \mathrm{mL}$ homogeneous $\mathrm{GO}$ aqueous dispersion;

(b) photographs of a 3DG after hydrothermal reduction at $180{ }^{\circ} \mathrm{C}$ for $12 \mathrm{~h}$;

(c) SEM images of the 3DG interior microstructures

Fig. 2 compares the XRD patterns of GO, 3DG, and graphite. For GO, a prominent peak of (001) faces exists at $10.99^{\circ}$, corresponding to an interlayer space of $8.05 \AA$. This peak disappears in the XRD pattern of 3DG, and a broad peak appears instead near the (002) peak of graphite at around $25^{\circ}$, which corresponds to a decreased interlayer space of $3.26 \AA$. This might be due to the $\pi-\pi$ stacking of graphene sheets during the 3DG assembly. Also, the broadness of the peak indicates a disordered orientation of graphene sheets in 3DG, which agrees the SEM results. 


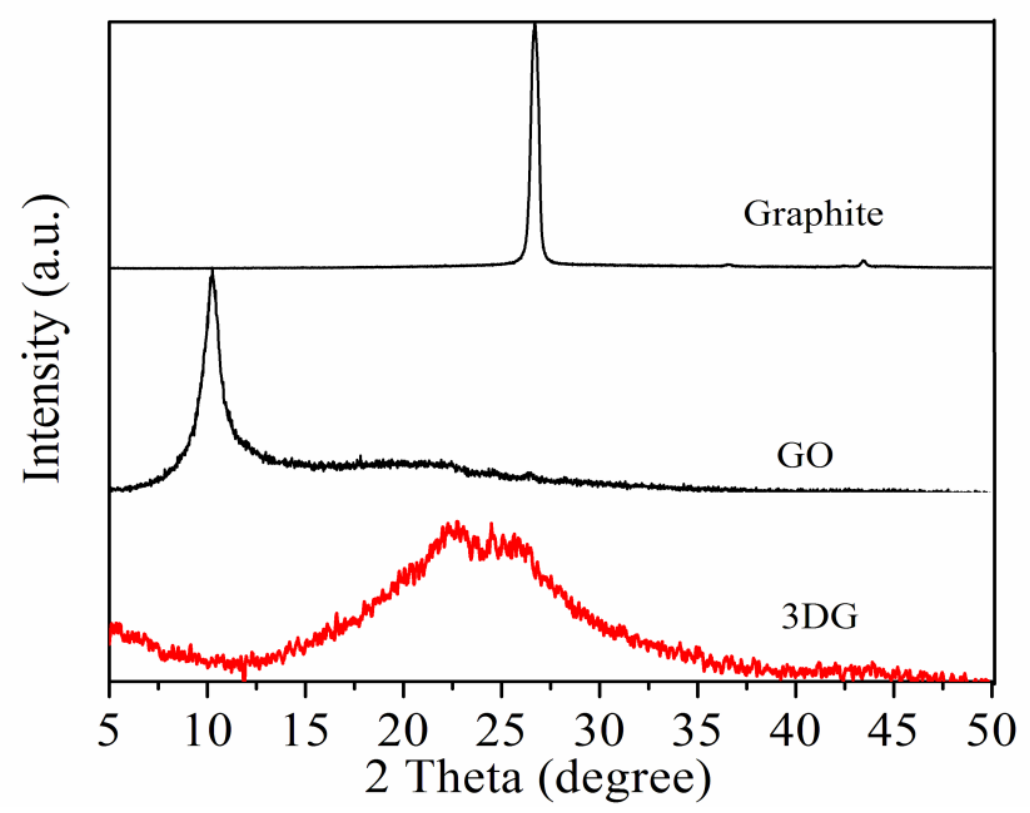

Fig.2 XRD patterns of natural graphite, GO, and freeze-dried 3DG.

In Fig.3, all Raman spectrum show the existence of the $\mathrm{G}$ band and D band, which are E2g vibrational mode in plane and A1g breathing mode, respectively. The binding of GO to GO sheets was confirmed by Raman spectroscopy. The tangential G Raman band of the GO sheets in 3DG $\left(1589 \mathrm{~cm}^{-1}\right)$ was found to be blue-shifted for about $15 \mathrm{~cm}^{-1}$ compared with those of pure GO and a graphite $\left(1586 \mathrm{~cm}^{-1}\right.$, Fig. 3). This phenomenon can be attributed to the strong interaction between the graphene layers in 3DG [8].

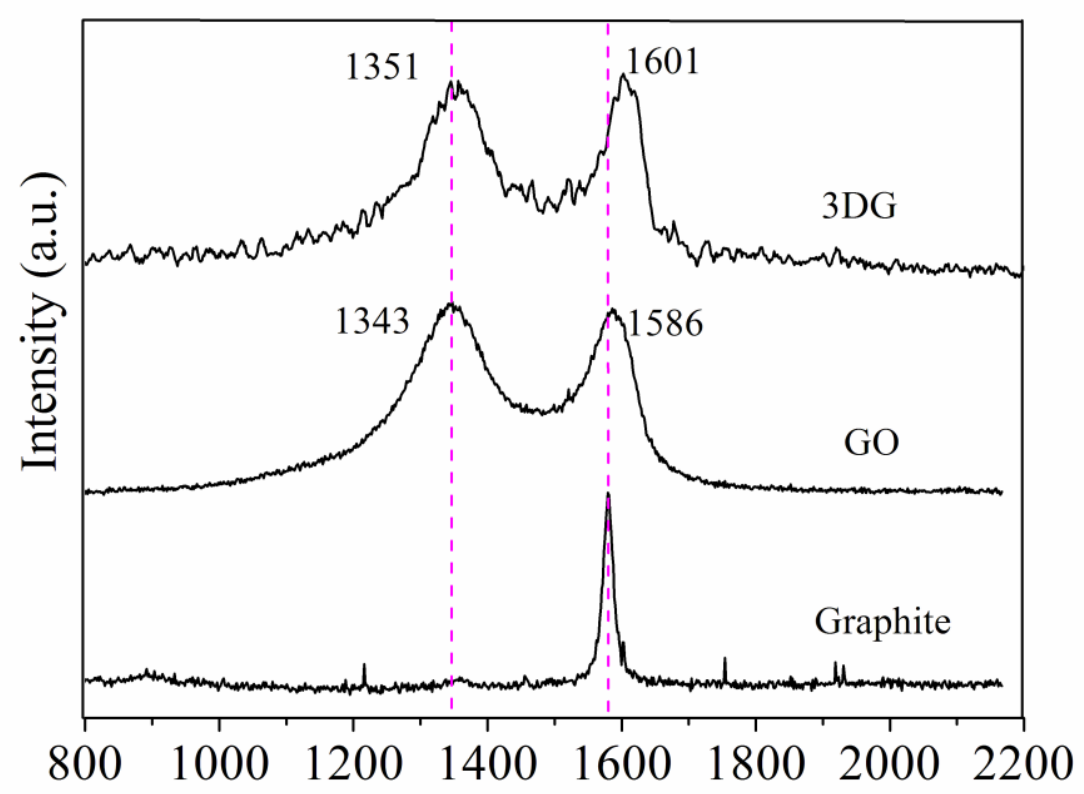

Fig.3 514 nm excited Raman spectra of graphite, GO, 3DG.

Fig.4 illustrated the $\mathrm{GO}$ concentration $\left(C_{\mathrm{GO}}\right)$ depended properties of the $3 \mathrm{DG}$. When $C_{\mathrm{GO}}$ was low $4 \mathrm{mg} / \mathrm{mL}$, only a black powdery material was produced after $12 \mathrm{~h}$ hydrothermal reduction. As $C_{\mathrm{GO}}$ was increased to $6 \mathrm{mg} / \mathrm{mL}$, mechanically stable 3DG samples were obtained. However, the size and strength of the former 3DG are not structurally stable. With the hand gently knead, it will be broken up. But when the $C_{\mathrm{GO}}$ was increased to 8 or $10 \mathrm{mg} / \mathrm{mL}$, the mechanically stable properties of $3 \mathrm{DG}$ are more stable than sample obtained at the $C_{\mathrm{GO}}$ of $6 \mathrm{mg} / \mathrm{mL}$. Before reduction, the $\mathrm{GO}$ sheets 
were randomly dispersed in water and in extended states, due to their strong hydrophilicity and electrostatic repulsion effect [9]. When GO sheets were hydrothermally reduced, they became regionally hydrophobic, due to their restored conjugated domains and diminished oxygenated functionalities as confirmed by the XRD results (Fig.2). The combination of hydrophobic and interactions caused a 3D random stacking between flexible graphene sheets. If $C_{\mathrm{GO}}$ was sufficiently high, the cross linking through partial overlapping of the flexible graphene sheets occurred timely and finally enough cross-linking sites were generated for forming a 3D network with pore sizes ranging from sub-micrometer to several micrometers (Fig. 1c).

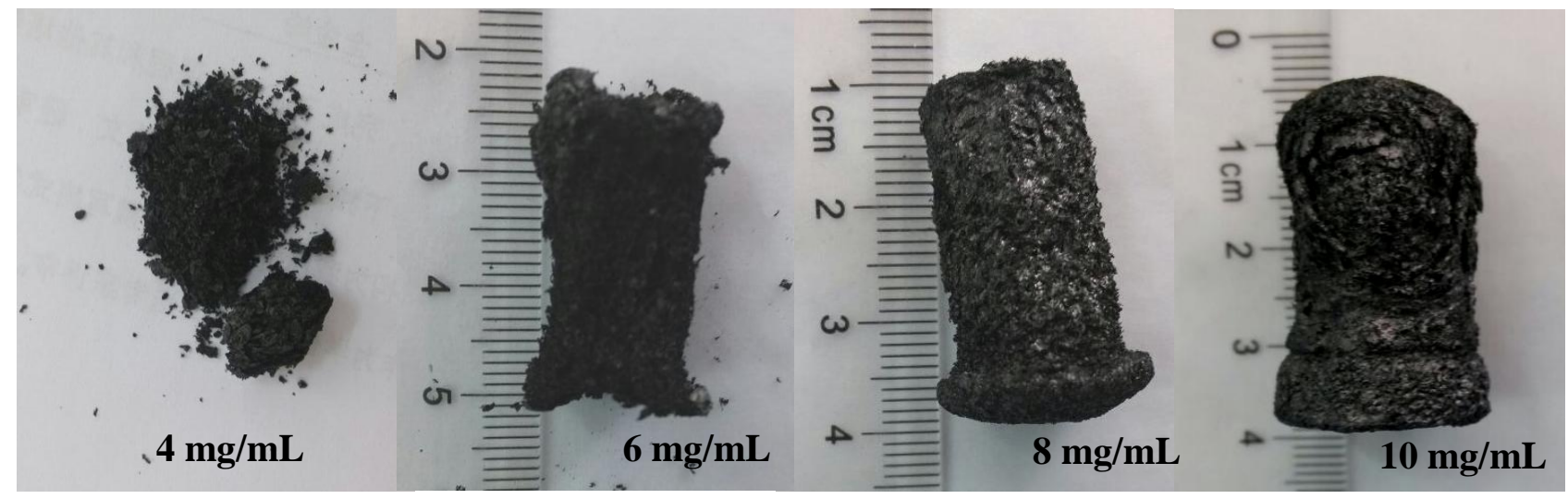

Fig. 4 Photographs of the products prepared by hydrothermal reduction of GO dispersions with different $C_{G O}$ at $180{ }^{\circ} \mathrm{C}$ for $12 \mathrm{~h}$

The progress of absorption is shown in a series of photos in Fig. 5 Photographs were taken every $1 \mathrm{~min}$. In this series $2 \mathrm{ml}$ soybean oil (stained with Congo red) was absorbed by 3DG block in $3 \mathrm{~min}$, 3DG appears as a dark sponge with the mass of $30 \mathrm{mg}$. The density of 3DG was calculated to be 13 $\pm 3 \mathrm{mg} \cdot \mathrm{cm}^{-3}$, which is comparable to or slightly lower than expanded graphite (EG) [10]. Conventional materials (especially EG) are stored in apertured containers (e.g. apertured plastic bags) before use; in contrast, 3DG can be molded into any shape, thus it could be placed directly in polluted areas and dredged intact afterward, making its recovery much simpler [11].

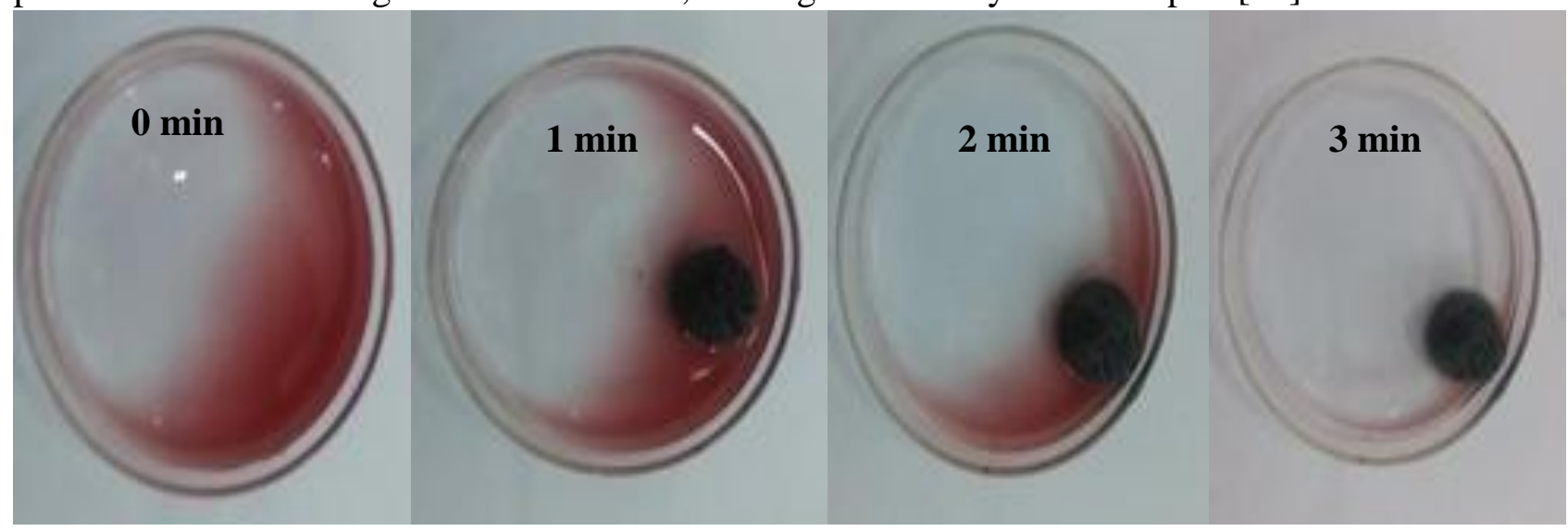

Fig.5 Photos of soybean oil absorption and characterization of 3DG.

Aother classes of organic liquids were evaluated in this study, e.g., commercial petroleum products engine oil and diesel oil with different carbon chain lengths, which are crucial components of crude oil. 3DG showed excellent absorption of these liquids ( Fig. 6a ). In general, 3DG could absorb the liquids at 20 to 56 times of its own weight. Its absorption efficiency is much higher than that of AC in the same adsorption time. The pore width distribution of 3DG (Fig. 6b) showed a wide distribution ranging from 2 to $60 \mathrm{~nm}$ which might be due to the randomly self-assembly process $[13,14]$. And the wide pore size distribution allowed 3DG has higher adsorption abilty. 

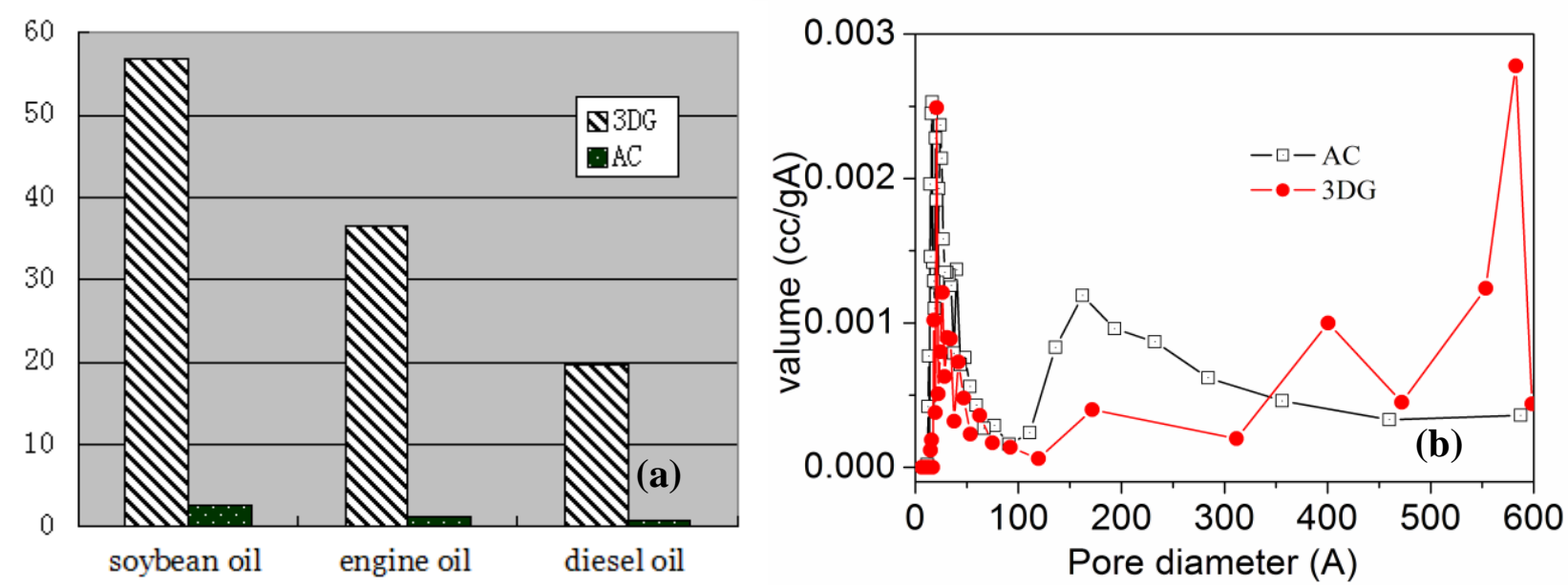

Fig.6 (a) Absorption efficiency of 3DG and AC; (b) adsorption pore diameter of 3DG and AC

\section{Conclusions}

In summary, we report an in-situ method to fabricate 3DG through the self-assembly solvent-thermal synthesis route without using any templates and additives. Due to the unique micro/nano-hierarchical morphology, the results show that 3DG has a strong potential to become a versatile, efficient, and safe absorber for hazardous organic liquids with applications in environmental protection and industrial production. This work provides a facile approach to control the synthesis of 3DG, and the processes to fabricate 3DG are facile, low-cost, and should be scalable to industrial levels.

\section{Acknowledgement}

This research was financially supported by the National Science and Technology Support Program of China (No. 2013BAE04B02 and No. 2013BAE04B03).

\section{References}

[1]I. Ali, V.K. Gupta, Advances in water treatment by adsorption technology, Nat. Protoc. 1 (2006) 2661-2667.

[2]S. Yin , Z. Niu , X. Chen, Assembly of graphene sheets into 3D macroscopic structures, Small. 8 (2012) 2458-2463.

[3]X. Xiao, T. E. Beechem, M. T. Brumbach, T. N. Lambert, D. J. Davis, J. R. Michael, C. M. Washburn, J. Wang, S. M. Brozik, D. R. Wheeler, D. B. Burckel, R. Polsky, Lithographically defined three-dimensional graphene structures, ACS Nano. 6 (2012) 3573-3579.

[4]C. Houa, Q. Zhang, Y. Li, H. Wang, P25-graphene hydrogels: room-temperature synthesis and application for removal of methylene blue from aqueous solution, J. Hazard. Mater. 205-206 (2012) 229-235.

[5]C. Li, G. Shi, Three-dimensional graphene architectures. Nanoscale. 4( 2012) 5549-5563.

[6]L. Sun, H. Yu, B. Fugetsu, Graphene oxide adsorption enhanced by in situ reduction with sodium hydrosulfite to remove acridine orange from aqueous solution. J. Hazardous Mater. 203204 (2012) 101-110.

[7]A. C. Ferrari, D. M. Basko, Raman spectroscopy as a versatile tool for studying the properties of grapheme. Nature Nanotech. 8 (2013) 235-246. 
[8]Y. Zhou, Q. L. Bao, L. A. L. Tang, Y. L. Zhong, K. P. Loh, Hydrothermal dehydration for the "green" reduction of exfoliated graphene oxide to graphene and demonstration of tunable optical limiting properties. Chem. Mater. 21 (2009) 2950-2956.

[9]Y. Xu, Q. Wu, Y. Sun, H. Bai, G. Shi, Three-dimensional self-assembly of graphene oxide and DNA into multifunctional hydrogels, ACS Nano. 4 (2010) 7358-7362.

[10]R. Chen, T. Zhao, J. Lu, F. Wu, L. Li, J. Chen, G. Tan, Y. Ye, K. Amine, Graphene-based three-dimensional hierarchical sandwich-type architecture for high-performance $\mathrm{Li} / \mathrm{S}$ batteries, Nano Lett. 13 (2013) 4642-4649.

[11]H. Bi, X. Xie, K. Yin, Y. Zhou, S. Wan, L. He, F. Xu, F. Banhart , L. Sun, R. S. Ruoff, Spongy graphene as a highly efficient and recyclable sorbent for oils and organic solvents. Adv. Funct. Mater. 22 (2012) 4421-4425.

[12]Y. Huang, C. Li, Z. Lin, EDTA-induced self-assembly of 3D graphene and its superior adsorption ability for paraquat using a teabag. ACS Appl. Mater. Interfaces. 6 (2014) 19766-19773.

[13]W. Lv, C. Zhang, Z. Li, Q. Yang Self-assembled 3D graphene monolith from solution J. Phys. Chem. Lett. 6 (2015) 658-668. 